\title{
Recent Developments in Integral Transforms, Special Functions, and Their Extensions to Distributions Theory
}

\author{
Adem Kılıçman, ${ }^{1}$ Mustafa Bayram, ${ }^{2}$ and Hassan Eltayeb ${ }^{3}$ \\ ${ }^{1}$ Department of Mathematics and Institute for Mathematical Research, University Putra Malaysia, \\ 43400 Serdang, Selangor, Malaysia \\ ${ }^{2}$ Department of Mathematical Engineering, Chemical and Metallurgical Faculty, Yildiz Technical University, \\ Esenler, 34210 Istanbul, Turkey \\ ${ }^{3}$ Department of Mathematics, College of Science, King Saud University, P.O. Box 2455, Riyadh 11451, Saudi Arabia
}

Correspondence should be addressed to Adem Kılıçman; kilicman@yahoo.com

Received 2 December 2013; Accepted 2 December 2013

Copyright (C) 2013 Adem Kılıçman et al. This is an open access article distributed under the Creative Commons Attribution License, which permits unrestricted use, distribution, and reproduction in any medium, provided the original work is properly cited.

The distributions are also known as generalized functions which generalize the idea of classical functions and allow us to extend the concept of derivative not only to all continuous functions but also to the discontinuous functions in the classical sense. The theory of distributions has applications in various fields especially in science and engineering where there are many noncontinuous phenomena which might naturally lead to differential equations whose solutions are distributions. For example, such as the delta distribution is not mathematical function in classical sense. However it is very useful in several applications, therefore the distributions can help us to develop an operational calculus in order to investigate linear ordinary differential equations as well as partial differential equations with constant and variable coefficients through their fundamental solutions.

By considering some regular operations which are valid for ordinary functions such as addition, multiplication by scalar scan is extended into distributions. Other operations can only be defined for certain restricted subclasses; these are also known as irregular operations. However, generalized functions are useful tools to extend the concept of derivatives and further to use them to formulate the generalized solutions of partial differential equations. They are also very important in physics and engineering where many noncontinuous problems naturally might lead to differential equations whose solutions are distributions, such as the Dirac delta distribution. This special issue is focused on some integrals transform, special functions, and their applications with fractional orders. In some papers, further relationship between linear and nonlinear partial differential equations and generalized functions was also considered.

We are pleased to announce the completion of this special issue on Recent Developments in Integral Transforms, Special Functions, and Their Extensions to Distributions Theory. This special issue was opened in late August of 2012 and closed in late May of 2013.

In this special issue, a total of 33 articles were published and they cover a wide range of special functions changes from theoretical sides of special functions to their applications in solutions of certain differential equations by mostly focusing on some special functions in several areas. There were also some submissions on the different types of integral transforms and their applications including some works on generalized functions. For example, the modeling of thermal distributions around a barrier at the interface of coating and substrate was studied by A. Sahin; A. Secer studied the numerical solution and simulation of second-order parabolic PDEs with Sinc-Galerkin method which covers to solve second-order PDEs by numerical method. Similarly, some integrals involving $q$-Laguerre polynomials and applications were studied by J. Cao.

An efficient pseudospectral method was reported to solve a class of nonlinear optimal control problems, which were presented by E. Tohidi et al. By M. Inc et al., numerical solutions of the second-order one-dimensional telegraph equation were studied which was based on the reproducing kernel 
Hilbert space method; in an interesting work by H.-L. Wu and J.-C. Lan, it was reported that Lipschitz estimates can be applied to fractional multilinear singular integrals on variable exponent Lebesgue spaces. In a paper by D. Kumar et al., an efficient approach for fractional harry dym equation by using Sumudu transform was presented. The stability of trigonometric functional equations in distributions and hyperfunctions was studied by J. Chung and J. Chang.

Further, by A. Atangana and A. Secer, the fractional order derivatives and table of fractional derivatives of some special functions were reported.

Since it would be very lengthy to list all the contributions, thus, we suggest that the readers read the full special issue to see the further details of each study.

\section{Acknowledgments}

The editors would like to express their gratitude to all the authors for their contribution and collaboration and to the many reviewers who were involved in the reviewing process and for their valuable comments, suggestions, and timely reports. Further we also acknowledge the assistance and help which were provided by the editorial board members of the journal during the preparation of this special issue as well as during the smooth publishing process. 


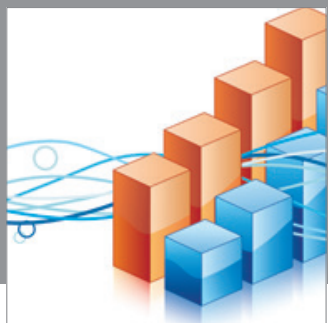

Advances in

Operations Research

mansans

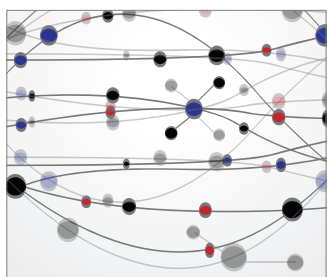

The Scientific World Journal
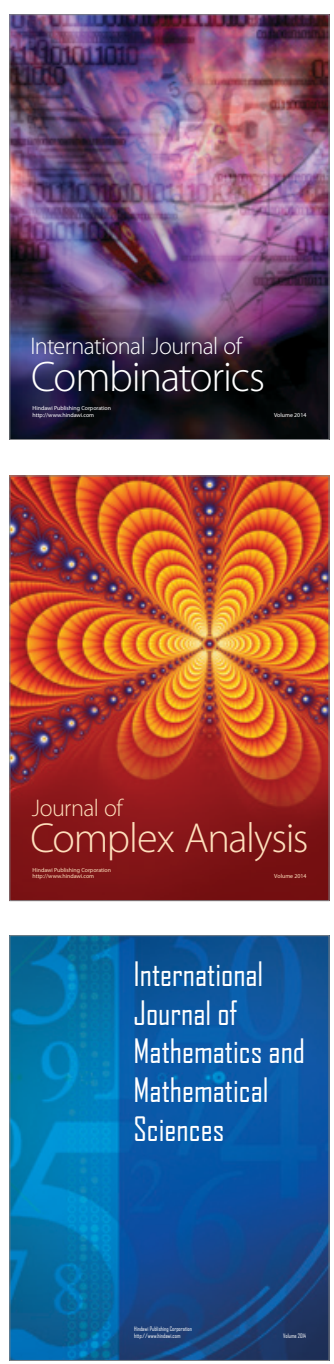
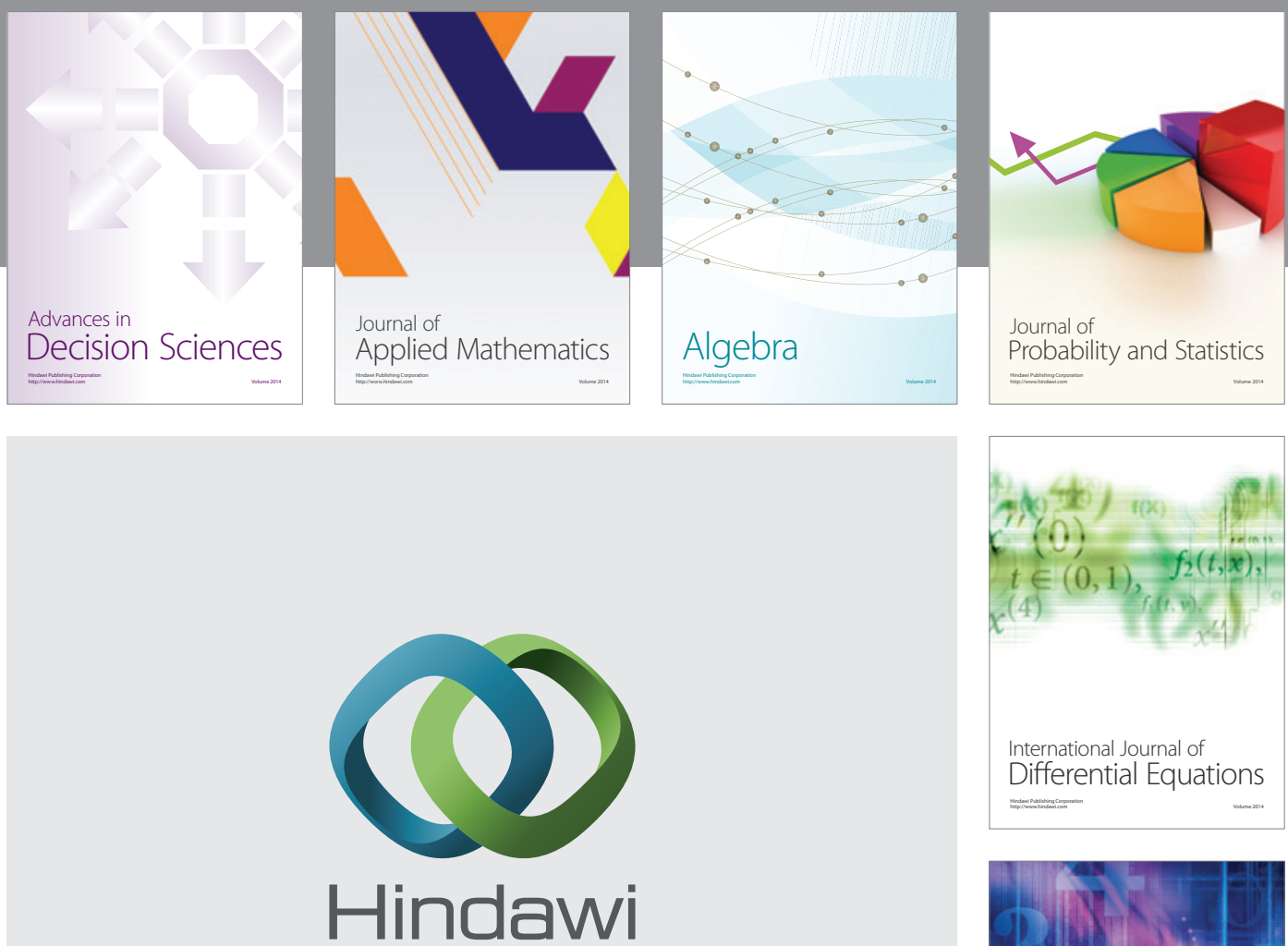

Submit your manuscripts at http://www.hindawi.com
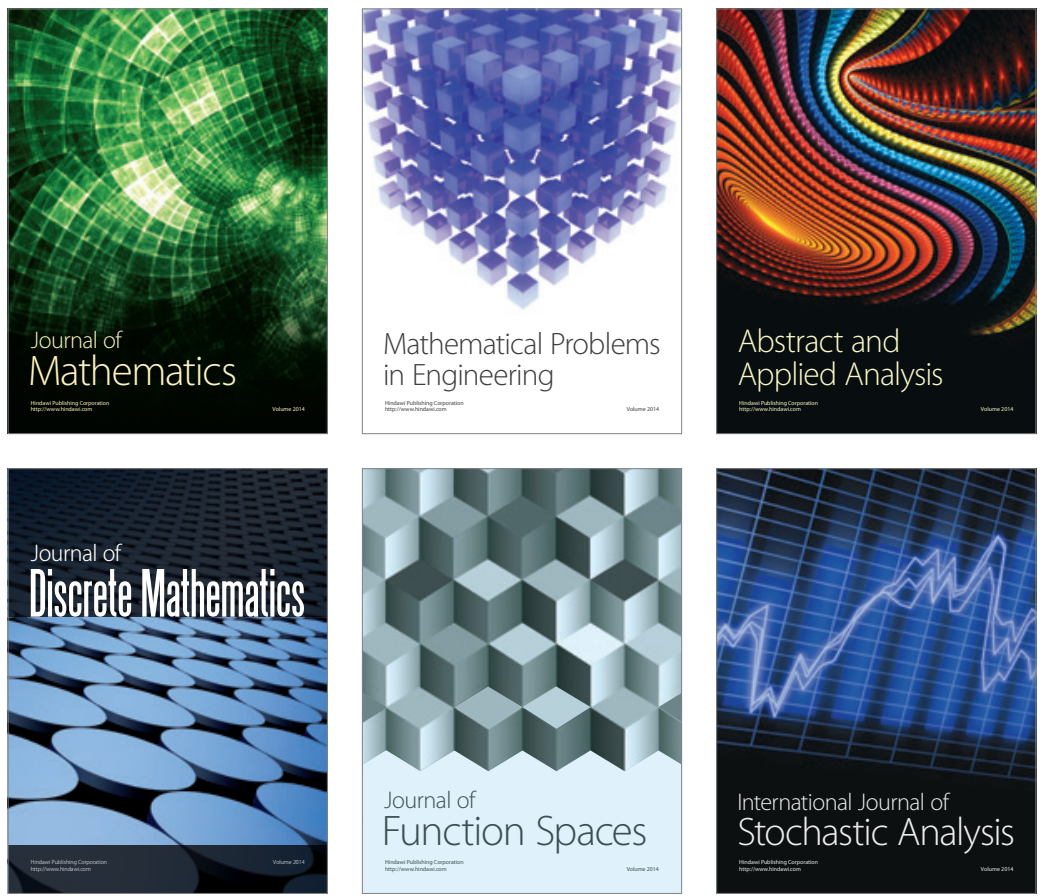

Journal of

Function Spaces

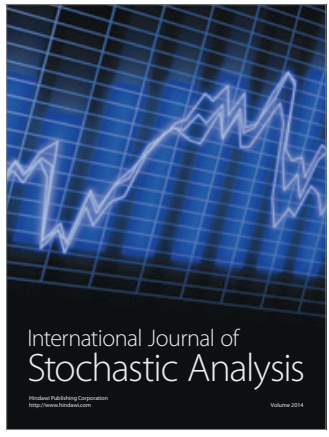

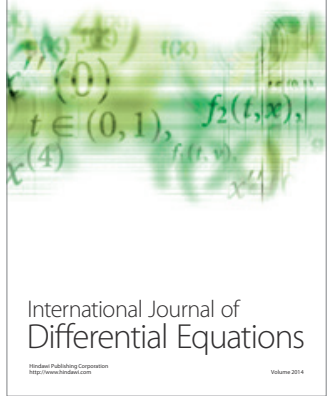
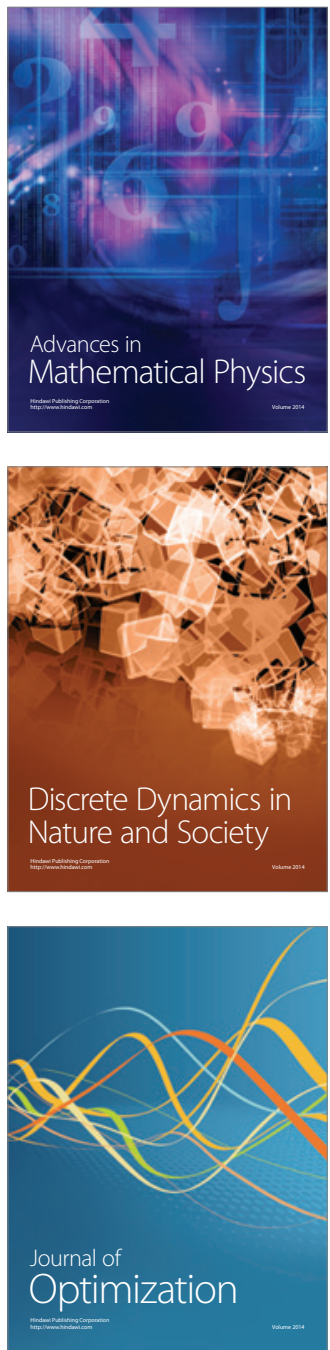